\title{
Late Gastric Incarceration 20 Years after Penetrating Chest Trauma
}

\author{
KeCheng Chen ${ }^{1}$ ChenHao Hsiao ${ }^{2}$ MingSung Yang ${ }^{2}$ \\ ${ }^{1}$ Department of Surgery, National Taiwan University Hospital Yun-Lin \\ Branch, Taiwan, Province of China \\ 2 Department of Surgery, Cheng Hsin General Hospital, Taipei, Taiwan, \\ Province of China \\ Address for correspondence ChenHao Hsiao, M.D., Attending \\ Physician, Department of Surgery, Cheng Hsin General Hospital, No.45 \\ Cheng-Hsin St, Taipei 11220, Taiwan, Province of China \\ (e-mail: oodaoda@gmail.com).
}

Thorac Cardiovasc Surg 2012;60(S2):e13-e15.

\begin{abstract}
Keywords

- diaphragm

- trauma

- empyema

The diagnosis of diaphragmatic injury in trauma patients is a challenge for surgeons. Misdiagnosis is common and can only be corrected when patients present with symptoms of diaphragmatic hernia. We report a rare case of delayed traumatic diaphragmatic hernia masquerading as empyema 20 years after lower chest penetrating trauma. The herniated stomach was found intraoperatively. Delayed traumatic diaphragmatic hernia should always be suspected in patients with trauma that may have occurred many years ago.
\end{abstract}

Traumatic diaphragmatic injury, which was first described by Sennertus in 1541, remains a diagnostic challenge for surgeons. ${ }^{1}$ It occurs in $\sim 3$ to $7 \%$ cases of abdominal or thoracic trauma. ${ }^{2}$ Traumatic diaphragmatic injury is caused by blunt traumas in $75 \%$ of the cases and by penetrating traumas in $25 \%$ of the cases. ${ }^{3}$ In penetrating traumas of the lower thoracic region, diaphragmatic injuries are observed in $15 \%$ cases of stab wounds and $46 \%$ cases of gunshot wounds. ${ }^{4}$ As traumatic diaphragmatic injuries are difficult to diagnose, a delayed diagnosis is common; the delay may extend from a few days to decades, even to 50 years. ${ }^{5}$ These injuries involve the herniation of abdominal contents into the thorax. The symptoms of delayed traumatic diaphragmatic hernia are mainly related to gastroenterological obstruction, such as nausea or vomiting. ${ }^{3}$ We present the rare case of delayed traumatic diaphragmatic hernia masquerading as empyema without any gastroenterological symptoms.

\section{Case Report}

A 55-year-old man presented with left chest pain and intermittent spiking fever for 2 days. The chest pain was aggravated on taking a deep breath or on changing the body position. He had dyspnea but no coughing, nausea, or vomiting. His previous medical history included penetrating trauma of the left posterior lower chest, which was caused by a screwdriver, complicated with hemothorax 20 years ago. At that time, he underwent chest tube insertion and then recovered without any operation.

At admission, the patient was $163 \mathrm{~cm}$ in height and $71 \mathrm{~kg}$ in weight. His temperature was $39.4^{\circ} \mathrm{C}$. His blood pressure was $120 / 90 \mathrm{~mm} \mathrm{Hg}$; pulse rate was 124 beats/min with sinus tachycardia; and respiratory rate was 24 breaths/min. Physical examination showed a decrease in breath sounds in the left lower chest with dullness on percussion. He had a leucocyte count of $20,800 / \mu \mathrm{L}$ with $84 \%$ neutrophils, hemoglobin level of $13.6 \mathrm{~g} / \mathrm{dL}$, and platelet count of $24.1 \times 104 / \mu \mathrm{L}$. Results of the liver function, renal function, and electrolyte tests were normal. A chest roentgenogram showed blunting of the left costophrenic angle (-Fig. 1). Chest sonography showed anechoic, complex, and nonseptated pleural fluid inside the enlarged cavity in the left lower chest (-Fig. 2). An ultrasound-guided thoracentesis was done, and muddybrownish, foul-smelling fluid was aspirated. Pleural fluid analysis showed a leucocyte count of $27,330 / \mathrm{uL}$ with $97 \%$ neutrophils, glucose concentration of $14 \mathrm{mg} / \mathrm{dL}$, lactic dehydrogenase (LDH) level of $3278 \mathrm{IU} / \mathrm{mL}$, and total protein concentration of $5 \mathrm{~g} / \mathrm{dL}$. The fluid was gram positive, and the culture was positive for Klebsiella pneumoniae and group D Enterococcus. The initial diagnosis was left-side empyema received

June 26, 2011

accepted after revision

July 12, 2011

published online

January 3, 2012 (c) 2012 Georg Thieme Verlag KG Stuttgart · New Yor
DOI http://dx.doi.org/ 10.1055/s-0031-1295582. ISSN 2194-7635. 


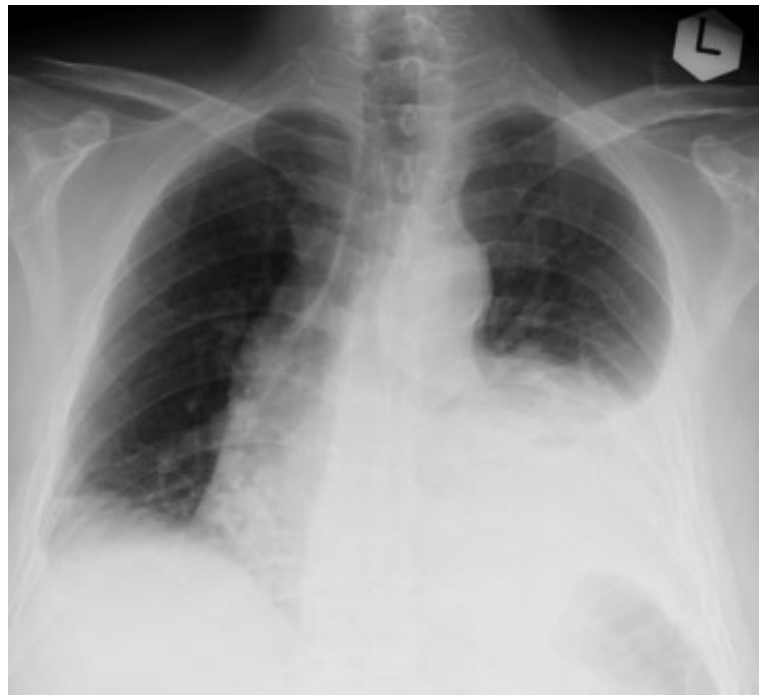

Figure 1 Chest roentgenogram showed left costophrenic angle blunting.

on the basis of the clinical symptoms, images, and the pleural fluid analysis. Owing to the patient's respiratory distress, we could not perform chest computed tomography. Due to his unstable and septic condition, he was referred to a thoracic surgeon for decortication.

The patient underwent left posterolateral thoracotomy instead of video-assisted thoracic surgery, because the patient could not tolerate one-lung ventilation. Much darkreddish turbid fluid inside the left chest cavity was removed. Then, a huge gangrenous stomach herniating into the left chest cavity was observed during the operation. The herniated stomach was resected via thoracoabdominal 55 stapler. A

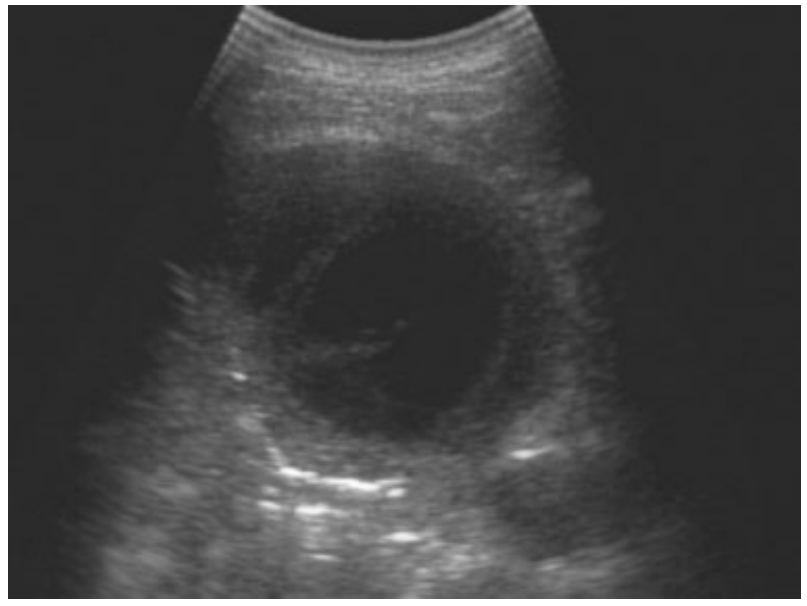

Figure 2 Chest sonography showed anechoic, complex, and nonseptated pleural fluid inside the enlarged cavity in the left lower chest.

substantial amount of turbid, foul-smelling fluid was observed within the gangrenous stomach. A 4-cm defect in the left hemidiaphragm was found after the resection of the strangulated stomach (-Fig. 3). The defect was primarily repaired with nonabsorbable sutures, and a chest tube was placed in the left chest cavity. The postoperative course was uneventful. He was discharged 18 days after the operation.

\section{Discussion}

Traumatic diaphragmatic injury is a recognized consequence of high-velocity blunt and penetrating trauma to the

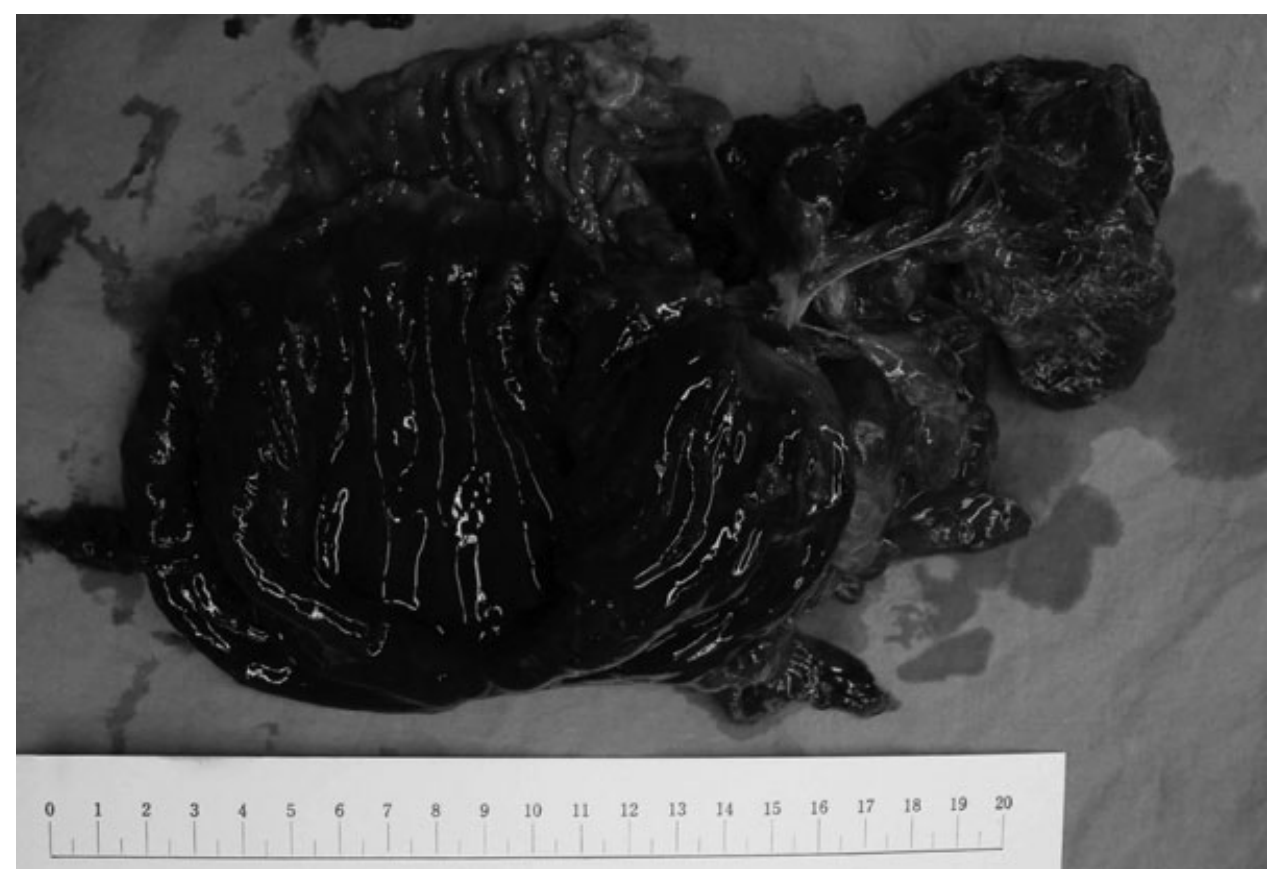

Figure 3 The opened, huge, dark-reddish strangulated stomach, $14 \mathrm{~cm}$ in maximal length, with blackish necrotic mucosae. 
abdomen or chest. ${ }^{6}$ However, delayed diagnoses are common in patients with minor traumas or multiple injuries. Patients may present with nonspecific symptoms and complain of chest pain, abdominal pain, dyspnea, tachypnea, and cough. ${ }^{7}$ In 1974, Grimes described the three phases of traumatic diaphragmatic injuries, namely, acute phase, latent phase, and obstructive phase. ${ }^{8}$ The acute phase extends from the time of original trauma to the diaphragm to the apparent recovery from the incurred injuries. Subsequently, the latent phase follows, wherein the intra-abdominal viscera occupy the defect and variably herniate into the thoracic cavity. The obstructive phase is complicated by the signs of visceral obstruction or strangulation, which manifest as herniation. Due to associated injuries, shock, respiratory insufficiency, coma, and the obscure nature of diaphragmatic injuries, the diagnosis of diaphragmatic injuries is frequently missed in the acute phase, and it is only diagnosed after presentation of obstructive symptoms. $^{3}$

The patient had a past medical history of penetrating trauma in the left lower chest. Traumatic diaphragmatic injury occurred and progressed to the latent phase wherein a portion of the stomach, supposedly the fundus, herniated into the chest cavity. The herniation probably enabled the smooth passage of food through the stomach. This was the reason that he did not have any obstructive symptoms of the gastrointestinal tract. After 20 years, the herniated stomach was enlarged and strangulated with turbid fluid inside. The diaphragmatic herniation was not suspected before because the patient did not present any gastroenterological symptoms. The sonographic pattern of empyema was mimicked by the herniated stomach. Ultrasound-guided thoracentesis was performed, and fluid analysis met the criteria for empyema, that is, leukocytosis, low $\mathrm{pH}(<7.20)$, low glucose concentration $(<60 \mathrm{mg} / \mathrm{dL}$ ), high $\mathrm{LDH}$, elevated protein levels, and the presence of gram-positive infectious organisms. Empyema was misdiagnosed according to clinical symptoms, findings of chest sonography, and analysis of thoracentesis.

In the first step of investigation, chest roentgenogram could diagnose $40.7 \%$ of diaphragmatic hernia. ${ }^{3}$ The diagnostic findings are gas bubbles in the chest, a nasogastric tube in the chest, irregularity of the diaphragmatic outline, elevated diaphragm, mediastinal shift without pulmonary or intrapleural causes, and compression atelectasis of the lower lobe. ${ }^{3,5}$ A gastrografin swallow X-ray, showing an outline of the esophagus and stomach, would also have resulted in the correct preoperative diagnosis of diaphragmatic hernia. ${ }^{3}$
Generally, chest computed tomography facilitates the diagnosis of diaphragmatic hernia. ${ }^{1}$ The common signs of diaphragmatic hernia on chest computed tomography are herniation of intra-abdominal organs into the hemithorax, absent diaphragm sign, and a defect of the diaphragm. ${ }^{1}$ Unfortunately, because of respiratory distress, the patient did not undergo gastrografin swallow X-ray and chest computed tomography before the operation. He underwent thoracotomy for empyema, but the strangulated stomach herniating into the pleural cavity was seen intra-operatively.

In conclusion, the diagnosis of diaphragmatic injury in trauma patients remains a challenge for surgeons. Lower chest and abdominal traumas are associated with high risks of diaphragmatic injury, especially in penetrating traumas. Delayed diagnosis of traumatic diaphragmatic injury can lead to traumatic diaphragmatic hernia after a long time. Surgical management is the only option for such patients. Irrespective of the symptoms presented, delayed traumatic diaphragmatic hernia should always be suspected in patients with a past medical history of lower chest or abdominal trauma that may have occurred many years ago. A gastrografin swallow X-ray and a chest computed tomography should be performed in these patients to exclude delayed traumatic diaphragmatic hernia.

\section{References}

1 Turhan K, Makay O, Cakan A, et al. Traumatic diaphragmatic rupture: look to see. Eur J Cardiothorac Surg 2008;33(6): 1082-1085

2 Reina A, Vidaña E, Soriano P, et al. Traumatic intrapericardial diaphragmatic hernia: case report and literature review. Injury 2001;32(2):153-156

3 Shah R, Sabanathan S, Mearns AJ, Choudhury AK. Traumatic rupture of diaphragm. Ann Thorac Surg 1995;60(5):14441449

4 Moore JB, Moore EE, Thompson JS. Abdominal injuries associated with penetrating trauma in the lower chest. Am J Surg 1980;140 (6):724-730

5 Rashid F, Chakrabarty MM, Singh R, Iftikhar SY. A review on delayed presentation of diaphragmatic rupture. World J Emerg Surg 2009;4;32

6 Goh BK, Wong AS, Tay KH, Hoe MN. Delayed presentation of a patient with a ruptured diaphragm complicated by gastric incarceration and perforation after apparently minor blunt trauma. CJEM 2004;6(4):277-280

7 Shreck GL, Toalson TW. Delayed presentation of traumatic rupture of the diaphragm. J Okla State Med Assoc 2003;96(4):181-183

8 Grimes OF. Traumatic injuries of the diaphragm. Diaphragmatic hernia. Am J Surg 1974;128(2):175-181 